\title{
Pautas de homofilia en Chile
}

\author{
Matías Bargsted \\ Instituto de Sociología \\ Pontificia Universidad Católica de Chile \\ mbargsted@uc.cl \\ Vicente Espinoza \\ Instituto de Estudios Avanzados (IDEA) \\ Universidad de Santiago \\ vicente.espinoza@usach.cl
}

\section{Alejandro Plaza}

Instituto de Sociología

Pontificia Universidad Católica de Chile

agplaza@uc.cl

\section{Resumen}

El presente artículo caracteriza los niveles de homofilia en Chile, es decir, la tendencia a que las relaciones sociales vinculen a personas socialmente similares. El estudio de esta propensión social permite comprender las bases de la cohesión y el conflicto en la sociedad actual. De una parte, la homofilia puede promover la integración social de personas en grupos sociales, pero, de la otra, puede favorecer relaciones cerradas y, con ello, la reproducción de una estructura social rígida y fragmentada. El análisis se basa en modelos log lineales aplicados a datos de redes personales (egocentradas) generados por medio de una encuesta nacional. Con un control por disponibilidad demográfica, los resultados muestran que las relaciones entre los chilenos están fuertemente estructuradas por la edad, la religión y, en un grado algo menor, por la posición política y el nivel educacional. También encontramos marcados niveles de variación intergrupal dentro de cada una de estas categorías sociales. En el caso de la posición política y la religión, la frecuencia de las relaciones sociales intragrupales es mucho menor entre los grupos mayoritarios que entre los grupos minoritarios. En suma, nuestras estimaciones apuntan a que en la sociedad chilena la clausura del grupo es una característica dominante, pero sujeta a variación según la categoría social considerada.

Palabras clave: homofilia; redes sociales; modelos log lineales; Chile 


\begin{abstract}
Patterns of Homophily in Chile
This article characterizes the levels of homophily in Chile, that is, the tendency for social relationships to connect people who are socially similar. The study of this social propensity helps understand the bases of cohesion and conflict in current society. On the one hand, homophily can promote social integration of people into social groups; but on the other, it can favor closed relations, and with it the reproduction of a rigid and fragmented social structure. Our analysis is based on log linear models applied to personal network data (ego-centered) captured in a national survey. The results show, while controlling for demographic availability, that social relations between Chileans are strongly structured by age, religion, and to a lesser degree, by political position and educational level. We also find large levels of intergroup variation within each of these social categories. In the case of political position and religion, the frequency of intra-group social relations is much lower among majority groups than among minority groups. In sum, our estimates suggest that a group closure logic is a dominant feature of Chilean society, but subject to variations depending on the social category considered.
\end{abstract}

Keywords: homophily; social networks; log linear models; Chile

\begin{aligned} & \multicolumn{2}{c}{ Sumario } \\ & 1. Introducción 6. Modelos de asociación \\ & 2. Aspectos teóricos respecto a la 7. Resultados estadísticos \\ & homofilia 8. Discusión de resultados y conclusiones \\ & 3. Antecedentes empíricos respecto a la Financiamiento \\ & 4. Medición de la homofilia Referencias bibliográficas \\ & 5. Resultados descriptivos Apéndice \end{aligned}

\title{
1. Introducción
}

La homofilia se refiere a la propensión de las personas a establecer relaciones con otras personas socialmente similares (Lazarsfeld y Merton, 1954; Marsden, 1988; McPherson et al., 2001). Esta similitud puede manifestarse en atributos como el sexo o la edad, el nivel socioeconómico o incluso en los estilos de vida, opiniones o creencias religiosas de las personas.

La relevancia sociológica de la homofilia se asocia con la posibilidad de caracterizar estructuralmente la cohesión e integración social. Las relaciones sustentadas por la homofilia producen una fuerte cohesión local que integra socialmente a las personas en grupos sociales, al facilitar su inserción en redes de apoyo y confianza (Hampton, 2016). Sin embargo, la homofilia supone también un problema, en la medida en que la cohesión del grupo puede reducir las interacciones externas y aumentar así la distancia con otros círculos sociales. El riesgo de fragmentación en la estructura social puede asociarse con fenómenos conflictivos, como la discriminación hacia minorías o la reproducción de la desigualdad social (Abascal y Baldassarri, 2015; Blau, 1977). Laumann 
y Senter (1976) argumentan incluso que el nivel de estratificación social en una sociedad puede definirse a partir del grado en que las personas de estatus social similar se eligen entre sí para establecer relaciones íntimas. En el límite, la homofilia puede producir una estructura social caracterizada por una alta integración local y una baja integración global (Marsden, 1988). En una línea similar, Lozares y Verd (2011) argumentan que estudiar las pautas de homofilia implica aproximarse de manera dinámica al estudio de la cohesión social. Esto en la medida en que las relaciones homofílicas serían el mecanismo por el cual las categorías sociales preexistentes se cristalizan en interacciones y relaciones sociales, y estas a su vez contribuyen a generar estructuras que producen nuevas o pretéritas identidades y categorías sociales.

La investigación empírica en torno a la homofilia en Chile y América Latina, aunque en creciente progreso, es relativamente incipiente. Los estudios previos se han concentrado en aspectos relativamente específicos, como los efectos negativos de la homogeneidad social en procesos de superación de la pobreza y la movilidad social (Espinoza y Canteros, 2001), las relaciones entre pares en población estudiantil (Ramos-Vidal et al., 2016; Valenzuela y Ayala, 2011) o en el desarrollo de redes de confianza y discusión política entre jóvenes (Ramos-Vidal et al., 2016). Otro aspecto desarrollado corresponde a los estudios sobre homogamia o el emparejamiento selectivo a partir de características como la educación, la raza y la ocupación (Rodríguez, 2016; Torche, 2010). No obstante estos avances, aún carecemos de investigación que aborde la homofilia de forma más global, es decir, que busque contrastar múltiples dimensiones sociales simultáneamente, que incluya diferentes tipos de lazos sociales y que abarque la población general de un país.

Esta ausencia de conocimiento requiere subsanarse por varios motivos. En primer lugar, el estudio empírico de la homofilia en Chile permitirá comprender mejor dos rasgos transversales de las sociedades latinoamericanas: la elevada desigualdad y la segregación social. En efecto, por medio del estudio de la homofilia, es posible determinar si en sociedades desiguales y segregadas como las latinoamericanas (Hoffman y Centeno, 2003) existe una sincronía entre procesos microsociales de interacción y la desigualdad o segregación a escala global. Segundo, aún persiste un claro desconocimiento acerca de cuáles son las dimensiones sociales que más potencian las relaciones sociales entre personas similares y, por ende, favorecen menor exposición a información social no redundante (Granovetter, 1973), lo que a su vez podría favorecer mayor polarización y fragmentación social en situaciones de conflicto social agudo. Y por último, en consideración a los cambios sociales económicos y políticos que las sociedades latinoamericanas están experimentado, la actual ausencia de registros empíricos impedirá, de no ser subsanada, conocer en el futuro la evolución temporal de los patrones de homofilia, como micromecanismo de la reproducción de las diferencias y desigualdades entre grupos demográficos (DiMaggio y Garip, 2011).

El presente estudio pretende analizar las pautas de homofilia y distancia social existentes en Chile. Para esto buscamos caracterizar y contrastar cómo 
influye la similitud social en la formación de redes sociales íntimas en cinco dimensiones socialmente relevantes: el género, la edad, el nivel educacional, la afiliación religiosa y las preferencias políticas. Además, intentaremos diferenciar las pautas de homofilia según si la relación entre entrevistados y confidentes es de tipo familiar o no, lo que permite ponderar la relevancia asignada por algunos autores a las relaciones familiares en las sociedades latinoamericanas (Torche y Valenzuela, 2011). Realizamos nuestro análisis empírico según modelos log lineales ajustados a datos de redes personales recabados por medio de un generador de nombres incluido en la encuesta nacional del año 2014 del Centro de Estudios de Conflicto y Cohesión Social (ENACOES).

El artículo comienza estableciendo algunas distinciones conceptuales respecto a la homofilia. A continuación, revisa descriptivamente los datos. Seguidamente, se caracterizan las pautas de asociación entre encuestados y confidentes, o egos y alteri, usando modelos estadísticos log lineales. Después de revisar los resultados empíricos, las conclusiones sintetizan las principales tendencias y proponen algunas líneas de investigación futura sobre este tópico para Chile y América Latina.

\section{Aspectos teóricos respecto a la homofilia}

Uno de los planteamientos conceptuales más influyentes para comprender los patrones de homofilia es la teoría macrosociológica de Peter Blau (1974; 1977; Blau y Schwartz, 1984). Para entender sus implicancias, comenzamos reseñando el concepto de integración social desarrollado por este autor. Siguiendo a Blau (1974), la integración social ocurre en dos niveles diferentes. En el nivel microsocial, la interacción social presencial y recurrente permite la integración de las personas a grupos sociales pequeños, por medio de la cual los individuos son socializados, desarrollan fuentes de apoyo social y crean interdependencias entre ellos. Este proceso describe cómo los individuos se convierten en miembros de grupos pequeños, como familias, camarillas o redes de amistad. A este nivel, los procesos de integración tienden a la homofilia, donde la formación de relaciones asocia personas con similar estatus o membrecía grupal y reduce el contacto entre miembros de diferentes grupos.

En contraste, en el nivel macrosocial, el proceso de inserción de los individuos y pequeños grupos sociales al conjunto de la sociedad está posibilitado por el nivel de heterogeneidad social, entendida como una función del número de grupos sociales y del tamaño relativo de los grupos en la población (Blau, 1974). ${ }^{1}$ Aunque suene paradojal, al aumentar el nivel de heterogeneidad de una sociedad, ya sea a través de la proliferación de nuevos grupos sociales o el

1. Siguiendo a Blau (1974), el nivel de heterogeneidad social aumenta al haber más grupos sociales, y si estos se distribuyen numéricamente de forma más uniforme. A modo de ejemplo, una sociedad dividida en tres grupos religiosos, donde uno incluye el $80 \%$ de la población, mientras que los otros dos contienen el $10 \%$ restante, sería menos heterogénea que otra sociedad dividida en cuatro grupos religiosos, donde cada cual contenga un $25 \%$ de la población. 
incremento en el tamaño relativo de uno o más grupos en la población, crece la frecuencia de relaciones sociales intergrupales. Esto ocurre al menos por dos razones. En primer lugar, en contextos sociales donde hay más grupos y estos se distribuyen de forma más uniforme, en simple virtud de las propiedades numéricas de los grupos, aumentan las oportunidades de contacto entre miembros de diferentes grupos sociales (Blau, 1977). Segundo, al ser mayor la heterogeneidad social, las demarcaciones grupales que delimitan a los miembros de un grupo se debilitan. Esto ocurre de forma particularmente visible en sociedades modernas caracterizadas por heterogeneidad multiforme, donde no hay una sola dimensión que diferencie a la población, sino múltiples ejes de diferenciación, como el religioso, el ocupacional, el étnico, el geográfico, el político u otros. Bajo esta estructura, cada individuo ocupa múltiples posiciones sociales, cada una de las cuales está asociada a una membrecía grupal diferente. De esta forma, la interacción social intragrupal en una dimensión (por ejemplo, nivel socioeconómico) puede ser simultáneamente un proceso de interacción social intergrupal en otra dimensión (como la denominación religiosa). La heterogeneidad multiforme promueve entonces que las personas tengan interacciones con miembros de otros grupos sociales porque favorece que la interacción intragrupal sea simultáneamente una forma de interacción intergrupal (Blau, 1974). ${ }^{2}$ En suma, contextos sociales más heterogéneos estimulan una mayor interacción social intergrupal, lo que favorece la integración macrosocial.

Los postulados de Blau implican que el estudio empírico de la homofilia debe necesariamente tener en cuenta el nivel de heterogeneidad social, ya que tanto la frecuencia de las relaciones intragrupales como intergrupales estaría condicionada, al menos en parte, por las oportunidades numéricas de contacto social. A modo de ejemplo, en una sociedad donde una amplia mayoría de la población se identifica con un grupo religioso en particular, digamos con la religión católica, la frecuencia de relaciones intragrupales entre católicos debería ser alta en simple virtud del hecho de que hay muchos católicos disponibles para establecer lazos. En contraste, en otra sociedad con múltiples grupos religiosos que gozan de similar tamaño poblacional, la frecuencia de relaciones entre católicos sería menor y, por ende, registraría un nivel de homofilia religiosa más bajo.

Siguiendo los planteamientos de Blau, en su influyente revisión sobre la literatura, McPherson et al. (2001) distinguen dos tipos de homofilia. Por un lado, la homofilia de base (baseline homophily) se refiere al patrón de vínculos de personas semejantes a partir de la demografía del conjunto de vínculos potenciales o, en otras palabras, del nivel de homofilia esperado bajo el supues-

2. Ahora, es importante notar que Blau especifica que este efecto de la heterogeneidad multiforme ocurre en la medida en que las posiciones sociales de las personas en diferentes dimensiones de diferenciación estén débilmente correlacionadas, o lo que denomina una estructura social intersectada. En contraste, si estas se encuentran correlacionadas, o lo que Blau llama consolidadas, las barreras sociales asociadas a diferentes membrecías grupales se adicionan y, por ende, es menos probable la interacción intergrupal. 
to de que la formación de lazos sociales fuese un proceso aleatorio determinado enteramente por el tamaño de los distintos grupos sociales. Por otro lado, la homofilia endogámica (inbreeding homophily) se refiere al sesgo positivo o negativo de las personas a formar lazos con otras personas socialmente similares - típicamente motivados por sus preferencias y gustos-, las cuales estarían determinadas, al menos parcialmente, por las posiciones sociales que ellas ostentan. La importancia de esta propensión endogámica debe determinarse necesariamente controlando por la homofilia de base.

Nuestro análisis de las pautas de homofilia en Chile considera cinco dimensiones de diferenciación social: género, edad, nivel educacional, afiliación religiosa y preferencias políticas. Siguiendo el lenguaje de Blau (1974; 1977), buscaremos determinar en qué medida estas variables de diferenciación constituyen "parámetros estructurales», es decir, distinciones sociales que se refieren a atributos sociales que las personas emplean y afectan de forma efectiva la formación y la composición de sus relaciones sociales.

\section{Antecedentes empíricos respecto a la homofilia}

Los estudios sobre homofilia realizados en Estados Unidos son de particular interés por su continuidad y la disponibilidad de resultados comparables con los de este artículo. En esta sociedad, la etnia o raza aparece como el principal factor discriminante de las relaciones con los confidentes en la red personal, incluyendo cónyuges (Kalmijn, 1998; Marsden, 1987; 1988). La edad también promueve patrones homofílicos asociados al ciclo vital, aunque se aprecia una reducción progresiva de ella con la transición hacia la longevidad. Si bien la homofilia por edad tiende a ser menor a la étnica, Marsden (1988) reporta que esta tiende a aumentar cuando no se consideran las relaciones de parentesco. Con respecto al género, la homofilia tiende a ser menor que en las otras características debido a que el número de hombres y mujeres es parecido en la sociedad.

Respecto a características adquiridas, la religión muestra altos grados de homofilia en instancias como el matrimonio, la amistad y las relaciones de confianza (Marsden, 1987; Kalmijn, 1998; Louch, 2000), y es particularmente fuerte en aquellos contextos con alta diversidad religiosa (McPherson et al., 2001). Variables como la educación, la ocupación y la clase social, según distintas investigaciones (Laumann y Senter, 1976; Louch, 2000; Marsden, 1987), fomentan que los vínculos entre egos y alteri sean más probables cuando estos comparten un estatus similar. De esta manera, y tal como lo expresan Laumann y Senter (1976: 1.313), los vínculos estarían motivados más por estar con «gente como uno» que por la búsqueda de estatus.

La investigación más reciente en Estados Unidos de Smith et al. (2014) muestra una pauta consistente de homofilia entre 1985 y 2004, con la raza y la religión como las principales dimensiones que estructuran la vinculación entre confidentes. También existiría una tendencia a la reducción leve de la homofilia, producto del aumento de heterogeneidad de la sociedad norteame- 
ricana en los últimos años, donde tener confidentes de otras religiones y razas se vuelve más probable en 2004 que en el año 1985.

Otras investigaciones relevantes sobre homofilia inducida en redes personales en Norteamérica y Europa muestran, en general, que incluso en el contexto de las mayores oportunidades de movilidad que ofrecen las sociedades modernas, la homofilia continúa siendo un rasgo preponderante (Blau y Schwartz, 1984; Brechwald y Prinstein, 2011; Ferrand et al., 1999; Forsé y Chauvel, 1995; Mollenhorst et al., 2008; Wright y Cho, 1992). Por ejemplo, en un estudio realizado en tres ciudades de Cataluña, en España, Lozares et al. (2014) reportan altos niveles de homofilia dentro de grupos de estatus de empleo. También observan altos niveles de homofilia por edad, no obstante, existen relaciones heterofílicas localizadas mayormente en las edades de alteri inmediatamente adyacentes a la edad de ego. Mollenhorst et al. (2008) hallan niveles elevados de homofilia en redes personales en Holanda, pero además muestran que la similitud social se acentúa cuando los lazos emergen en algunos contextos sociales particulares, como la escuela o el trabajo. La evolución de la homofilia en Francia muestra una reducción en términos de origen social u ocupación, aunque se mantiene relativamente estable en términos de niveles de educación y riqueza (Forsé y Chauvel, 1995).

El estudio de la homofilia es una línea de investigación en progresivo desarrollo en América Latina. Ramos-Vidal et al. (2019) estudió la homofilia para caracterizar el nivel de cohesión social en el contexto de localidades y comunidades fuertemente afectadas por violencia política. Espinoza y Canteros (2001) abordaron los efectos negativos de la homogeneidad social en procesos de superación de la pobreza y la movilidad social. Otro conjunto de investigadores han considerado patrones de homofilia en población juvenil para estudiar popularidad y relaciones entre pares en el aula (Ramos-Vidal, 2016), el efecto par sobre la iniciación del uso de drogas (Valenzuela y Ayala, 2011), y las redes de confianza y discusión política en el contexto de organizaciones sociopolíticas (Ramos-Vidal et al., 2016). Finalmente, algunos estudios han abordado los niveles de homogamia matrimonial en América Latina (Gullickson y Torche, 2014; Solís, 2010; Torche, 2010). Estos estudios han establecido la fuerte similitud en las características sociales de los cónyuges, y han destacado la relevancia de la educación en el proceso de selección de parejas. Según Torche (2010), la homogamia educacional observada en parejas chilenas se ubica en niveles cercanos al $50 \%$, lo cual es semejante al nivel de Estados Unidos, pero menor al nivel de Brasil.

De acuerdo con los antecedentes teóricos y empíricos reseñados arriba, derivamos dos hipótesis muy sencillas que guían nuestro análisis del caso chileno. En primer lugar, y controlando por disponibilidad demográfica, esperamos observar un patrón de interacción social predominantemente endogámico, es decir, donde la interacción social entre miembros de un mismo grupo social será más frecuente que entre personas de distintos grupos (hipótesis 1). Segundo, y para el caso específico de las dimensiones que pueden asociarse a diferencia de graduaciones (nivel educacional y edad), la frecuencia de las relaciones 
sociales entre miembros de distintos grupos sociales se reducirá a medida que la distancia social entre personas se acreciente (hipótesis 2).

\section{Medición de la homofilia}

Para estudiar los niveles de homofilia en las relaciones sociales de los chilenos, empleamos los datos de la Encuesta de Conflicto y Cohesión Social en Chile (ENACOES) de 2014, realizada por el Centro de Estudios del Conflicto y la Cohesión Social (COES). Esta encuesta empleó un diseño muestral probabilístico multietápico por conglomerados. Se entrevistaron personas de entre 18 y 75 años, elegidas aleatoriamente dentro de cada hogar. La muestra se aplicó en 75 comunas del país y es representativa del 78,1 \% de la población urbana y el 68,0 \% de la población del país. ${ }^{3}$ Los datos de la encuesta fueron procesados considerando el efecto de diseño y ponderados para corregir las probabilidades de selección de los entrevistados, así como la distribución poblacional del sexo y la edad de los entrevistados, mediante el paquete survey de R (Lumley, 2017).

La encuesta incluyó una batería de redes sociales egocéntricas, comúnmente llamada generador de nombres, a partir de la cual se pueden caracterizar los ambientes interpersonales de los encuestados (Burt, 1984; Marsden, 1990). A cada encuestado se le aplicó la siguiente pregunta: Ocasionalmente la gente conversa de asuntos que le importan con otras personas. Pensando en los últimos seis meses, ¿quiénes son las personas con las que usted ha conversado?t Después de que los encuestados indicaran los nombres de pila de hasta un máximo de cinco personas con quien conversaron, o lo que denominamos sus confidentes, se les consultó acerca del género, la edad, la educación, la religión y la posición política de las personas nombradas. ${ }^{5}$ El límite al número máximo de posibles confidentes se tomó siguiendo las recomendaciones de Burt (1984), que buscan conciliar un número suficiente de confidentes para poder capturar heterogeneidad en el entorno social de los encuestados, y el tiempo que con-

3. La metodología del estudio y la base de datos se encuentran disponibles en https://dataverse. harvard.edu/dataset.xhtml?persistentId=doi:10.7910/DVN/SRPWFW

4. Este tipo de pregunta tiende a generar integrantes de la red que poseen una relación cercana con el respondiente, por lo cual se les denominará confidentes (Marin, 2004). No obstante, la formulación de la pregunta no establece ninguna restricción en cuanto a qué es lo que el respondiente considere importante. Empleamos el criterio de temas importantes siguiendo el diseño desarrollado por Burt (1984) para la General Social Survey aplicada en Estados Unidos. Un generador puede usar muchos otros criterios o roles (por ejemplo, personas con quienes se habla de temas políticos o proveen ayuda material). Marsden (1990: 442) provee una discusión más detallada sobre el tópico.

5. Puede llamar la atención en nuestro análisis la ausencia de una dimensión étnica o racial, que constituye un principio estructurante relevante en otros contextos (Abascal y Baldassarri, 2015; McPherson et al., 2001). En el caso chileno, las principales diferencias étnicas están asociadas históricamente con la pertenencia a pueblos indígenas y más recientemente a grupos migrantes. La omisión de esta dimensión social se debe a que este descriptor de los contactos está ausente en la encuesta. Queda pendiente abordar esta dimensión en una investigación futura. 
lleva la aplicación de este instrumento en el contexto de encuestas generales de opinión pública. ${ }^{6}$

Las preguntas empleadas para medir las características de los encuestados y sus confidentes fueron equivalentes o muy similares. La edad se midió solicitando la edad exacta del encuestado y sus confidentes, al igual que el sexo. El nivel educacional se midió para ambos casos usando una pregunta sobre el nivel educacional alcanzado con 10 categorías de respuesta, cuyo rango varía de $\operatorname{Sin}$ estudios a Postgrado, Magister, Doctorado. Las respuestas fueron posteriormente recodificadas en cuatro tramos: educación básica, educación media (secundaria), educación superior técnica y educación superior universitaria. La posición política de los encuestados fue consultada usando una pregunta de autoubicación ideológica en una escala numérica con rango de 1 (izquierda) a 10 (derecha). Los valores 1 al 4 fueron categorizados como izquierda, 5 y 6 como centro, y 7 o más como derecha. Adicionalmente, todas las personas que mencionaron No sabe o No responde fueron clasificadas como Independiente o No sabe. En cambio, la posición política de los confidentes fue registrada por medio de la siguiente pregunta: En términos de identificación ideológica, ¿[NOMBRE] es una persona de derecha, centroderecha, centro, centroizquierda o izquierda? El encuestado también podría mencionar Ninguna, No sabe o No responde, y todas estas respuestas se clasificaron como Independientes o No sabe. Las respuestas derecha y centroderecha (centroizquierda o izquierda) fueron categorizadas como Derecha (Izquierda). Finalmente, la religión de los encuestados se midió preguntando por la religión o Iglesia a la que pertenece o se siente más cercano, mientras que para los confidentes se les preguntó si conocen ¿cuál es la creencia religiosa con la que se identifica o siente más cercano [NOMBRE]. En ambos casos, se recodificaron las respuestas en las cuatro categorías: Católicos, Evangélicos (que incluyen pentecostales), Otra religión e Irreligiosos. Los estadísticos descriptivos de todas estas variables están disponibles en el apéndice (sección A).

\section{Resultados descriptivos}

En total, hay 1.884 encuestados con al menos un integrante en su red de conversación, cuyos contactos alcanzan 5.810 personas. ${ }^{7}$ El tamaño medio

6. Burt (1984) asume que hay cierto nivel de correlación entre el nivel de cercanía o intimidad con los confidentes, capturado por el orden de mención de los alteri, y la similitud social entre los encuestados y confidentes. Bajo este supuesto, limitar el número de posibles confidentes disponibles para mencionar podría sesgar la caracterización del entorno interpersonal a favor de una mayor homogeneidad.

7. Analizamos el porcentaje de díadas perdidas debido a respuestas No sabe / No responde en el reporte de la edad, educación y religión de los alteri (el sexo tiene datos completos, y en la posición política la alternativa No sabe / No responde fue fundida con la categoría de Independientes). Para edad se reporta un $5 \%$ de no respuestas, para religión un 10,9\%, y para educación un $13 \%$. Encontramos diferencias significativas $(\mathrm{p}<0.01)$ en la tasa de no respuesta según el tamaño de la red de ego, no obstante, no son gran magnitud. En el caso más extremo - la religión-, las diferencias van desde un $4 \%$ entre los encuestados que mencionan a dos confidentes hasta un $13 \%$ entre aquellos que mencionan a cinco confidentes. 
de la red en esta muestra es de 3,1 integrantes $(\mathrm{DE}=0,09)$. Un cuarto de la muestra $(25 \%)$ mencionó un solo confidente, mientras que más de la mitad de los encuestados $(52,1 \%)$ registran al menos tres personas con las que pueden discutir temas importantes. Un porcentaje menor de los entrevistados mencionaron no tener confidentes $(7,1 \%)$ y fueron excluidos del análisis. Este resultado es bastante similar a lo reportado por McPherson et al. (2006) para el caso norteamericano en 1985, donde el nivel de aislamiento social - $\mathrm{O}$ personas que no reportan confidentes-alcanza al $8 \% .^{8}$

En el gráfico 1, se representan las tablas de contingencia que cruzan el sexo, la edad, la educación, la religión y la posición política de los encuestados y sus confidentes. ${ }^{9}$ Cada cuadrante representa el porcentaje de díadas encuestadoconfidentes, o más genéricamente ego-alteri, respecto al total de encuestados observados. Dado que algunos encuestados mencionaron más confidentes que otros, las tablas le dan implícitamente mayor peso a los encuestados con redes de mayor tamaño, lo cual plantea el riesgo de que los resultados del análisis sean producto de la construcción de las tablas. Análisis complementarios no encuentran una asociación significativa entre las pautas de homofilia y el tamaño de las redes. ${ }^{10}$

En términos generales, se puede observar que en las cinco variables existe una alta proporción de casos en las diagonales (colores más oscuros), lo que indica una clara propensión hacia la endogamia grupal en las relaciones sociales de los chilenos. Las tendencias más extremas se observan en la religión y la posición política. Del total de confidentes mencionados por los encuestados católicos, un $84 \%$ corresponde a otras personas católicas. Los porcentajes respectivos descienden entre los encuestados de otras religiones, pero aún son relativamente altos. Al considerar la posición política, observamos el porcentaje de interacción intragrupal más alto: el $91 \%$ de los confidentes de los

8. Cabe destacar que en el año 2009 se concitó una polémica respecto a los resultados de McPherson et al. (2006), quienes reportan que el aislamiento social creció en Estados Unidos entre 1985 y 2004 desde un 8 \% hasta un $23 \%$. Para Fischer (2009), los resultados obtenidos por la GSS de 2004 no tienen correlato con procesos sociales sustantivos, y podrían constituir un artefacto metodológico motivado por fatiga de los encuestados, entre otras razones. En su réplica, McPherson et al. (2009) pudieron dar cuenta de algunos elementos presentados en la crítica de Fischer, pero mediciones independientes que han utilizado el mismo generador de nombres no han podido replicar el nivel de aislamiento social encontrado inicialmente en 2004 (Hampton et al., 2011).

9. Ver en el apéndice (cuadro 2A) las tablas de contingencia representadas en el gráfico 1, que son empleadas posteriormente para calcular los modelos log lineales.

10. Se evaluaron modelos multinivel multinomiales bayesianos que predicen la membrecía grupal de los alteri (variable dependiente nominal) según las categorías de membrecía grupal de ego (variable independiente). En estos modelos, los alteri mencionados están anidados en los encuestados. Estimamos dos conjuntos de modelos: uno que incluye el tamaño de la red cercana como variable de control y otro que la excluye. Los resultados están disponibles en el apéndice B. Ahí se puede apreciar que los coeficientes asociados a las categorías de membrecía grupal de ego entre ambos modelos son muy similares y estadísticamente indistinguibles. Esto indica que las pautas de asociación no están influenciadas por el tamaño de la red cercana de los encuestados. 
Gráfico 1. Díadas ego y alter que discuten asuntos importantes, por sexo, edad, educación, religión y posición política. Los porcentajes son calculados según el total de cada fila (datos ponderados)
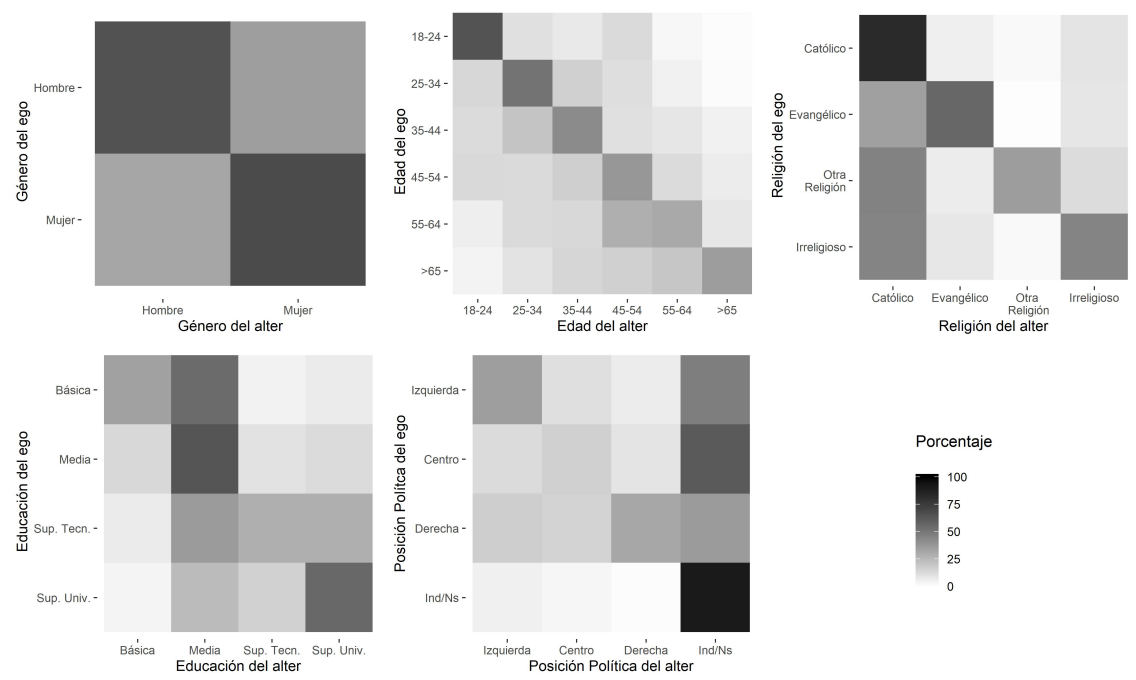
Religión del alter

Fuente: elaboración propia según ENACOES 2014.

encuestados que se categorizan como Independientes o No sabe pertenecen al mismo grupo político. También es posible notar porcentajes elevados entre encuestados que declaran posiciones tradicionales (particularmente aquellos de centro) y confidentes independientes. Esto se debe a que una gran mayoría de los confidentes (63\%) son clasificados como Independientes o No sabe por los encuestados, lo que presumiblemente refleja el bajo interés que los chilenos manifiestan por los asuntos políticos (Luna y Altman, 2011; Silva, 2004).

Los patrones de asociación según edad y educación también indican un patrón endogámico, con un $44 \%$ y un $51 \%$, respectivamente, del total de las díadas, y se ubican en la diagonal de cada tabla. Pero además es posible distinguir un interesante patrón complementario. En ambas variables, decrece la proporción de díadas entre encuestados y confidentes a medida que las diferencias etarias o educativas aumentan. Esto se evidencia más claramente en las columnas de los extremos, que progresivamente se vuelven más claras, ya sea en dirección descendente (para los encuestados de 18 a 24 años o los con educación básica) o ascendente (para los encuestados de 65 años o más, o los con educación superior universitaria).

Estos patrones, si bien pueden ser ilustrativos, tienen una importante limitación: los niveles de asociación entre los encuestados y sus confidentes mezclan la propensión de las personas a formar lazos (homofilia inducida) con otras personas de un mismo grupo social con la disponibilidad numérica de esas personas en la población (homofilia de base). En la dimensión religiosa y política, 
un miembro de un grupo social numeroso, como los encuestados católicos o independientes, tiene mayor probabilidad de establecer un lazo social endogámico en simple virtud de que hay muchas personas con igual membrecía.

\section{Modelos de asociación}

Para capturar de un modo más preciso los patrones de asociación en las relaciones sociales cercanas de los chilenos empleamos modelos log lineales. Por medio de estos, buscamos estimar, a través de un modelo lineal generalizado con distribución Poisson, el número de casos observados en las celdas de las tablas de contingencia representadas gráficamente en la sección anterior.

En contraste con las simples tablas bivariadas, los modelos log lineales, tal como menciona Marsden (1988) y Smith et al. (2014), nos permiten modelar los patrones de asociación de las díadas entre encuestados y confidentes controlando por la distribución marginal de cada variable, esto es, por el tamaño relativo de cada grupo en la población. A modo de ejemplo, se estima la frecuencia esperada de díadas entre personas con igual afiliación religiosa controlando por la cantidad de encuestados y confidentes que se identifican en cada una de las posibles categorías religiosas incluidas en el análisis. ${ }^{11}$

Existe una variedad de modelos que pueden ajustarse a los datos de las tablas de contingencia. A continuación, presentamos los modelos estimados proveyendo el correlato sociológico acerca de cómo se estructuran las relaciones entre personas. Todos los modelos estimados son casos especiales de la siguiente ecuación:

$$
\log \mu_{i j}=\lambda+\lambda_{i}^{E}+\lambda_{j}^{C}+\delta_{k}+v_{i j}(1)
$$

donde $\mu_{i j}$ representa el número esperado de díadas entre encuestados (E) y confidentes (C) en la celda $i j$ de la tabla, los parámetros $\lambda_{i}^{E}$ y $\lambda_{j}^{C}$ representan los efectos de fila y columnas asociados a cada categoría de membresía grupal de encuestados y confidentes, el parámetro $\delta_{k}$ captura posibles efectos de endogamia grupal localizados en la diagonal de las tablas de contingencia, y el parámetro $v_{i j}$ captura efectos interactivos asociados a encuestados y confidentes que pertenecen a distintos grupos.

El primer modelo que consideramos es el Modelo de independencia, que asume que el número de díadas encuestados-confidentes observado en cada celda es una función solamente de $\lambda_{i}^{E}$ y $\lambda_{j}^{C}$, la cantidad total de casos en cada fila y columna correspondientes a las categorías de la variable de membresía grupal. En términos de

11. Los modelos de regresión multinivel aplicados a datos egocéntricos (tal como los reseñados en el apéndice B o empleados en otros estudios como el de Miguel Luken y Tranmer, 2010) no controlan por la disponibilidad numérica de egos en la población. Si bien tienen la ventaja de poder incorporar múltiples predictores (incluidos atributos de los alteri), y corregir por la falta de independencia en las observaciones sobre los lazos observados, hemos optado por el empleo de modelos log lineales, dada que nuestra prioridad teórica es diferenciar la homofilia inducida de la homofilia de base. 
la ecuación (1), esto implica que se constriñe a que $\delta_{k}=0$ y $v_{i j}=0$. Este modelo presume que la relación entre las características de los encuestados y de sus confidentes es estadísticamente independiente y, en consecuencia, las díadas se forman solamente según las oportunidades determinadas por el tamaño de cada grupo. El modelo de independencia no tiene valor teórico propio, pero cumple una función primordial como un punto de contraste de los modelos que detallamos a continuación.

La segunda especificación se refiere al Modelo de endogamia constante (EC) y Endogamia diferenciada (ED). Este modelo agrega al modelo de independencia el parámetro asociado a la interacción entre los valores de ego y alter cuando ambos son miembros de un mismo grupo social $(i=j)$. Cuando se constriñe este parámetro a un mismo valor para todos los grupos $\delta_{k}=\delta$, se obtiene el modelo de endogamia constante, mientras que el modelo de endogamia diferenciada, también llamado Modelo de cuasiindependencia, permite que el nivel de asociación varíe para cada uno de los grupos. Ambos modelos presumen que las relaciones intergrupales son estadísticamente independientes y, en consecuencia, el efecto de una mayor o menor disimilitud social no afecta la frecuencia de relaciones entre un ego y alter (es decir: $v_{i j}=0$ ).

En tercer lugar, tenemos el Modelo de cuasisimetría, que extiende el modelo de endogamia diferenciada al presuponer que la propensión a establecer relaciones entre un ego y alter que pertenecen a distintos grupos sociales es equivalente a la propensión a establecer relaciones entre un ego y alter que pertenecen a las categorías invertidas. En términos estadísticos, esto implica que $v_{i j}=v_{j i}$. A modo de ejemplo, la propensión de los encuestados católicos a tener relaciones cercanas con confidentes evangélicos sería equivalente a la propensión de los encuestados evangélicos a tener relaciones cercanas con confidentes católicos. En términos sociológicos, se presupone que los niveles de interacción social entre miembros de distintos grupos sociales son recíprocos y que no hay discriminaciones grupales unilaterales.

El cuarto modelo se refiere al Modelo de asociación uniforme con endogamia constante y diferenciada. Este incorpora una interacción lineal entre las categorías de respuesta de cada variable grupal de los encuestados y confidentes bajo el supuesto de que son de carácter ordinal y, por ende, pueden ser asignadas a valores numéricos enteros. En términos de la ecuación (1) esto implica: $v_{i j}=\beta_{E C}$. En el presente estudio esto se aplica para la edad y educación. El parámetro interactivo $\beta_{E C}$ permite evaluar si la frecuencia de relaciones intergrupales decrece a medida que aumenta la distancia social. Estimamos este modelo incluyendo efectos de endogamia constante y diferenciada.

La última especificación se refiere al Modelo de parámetros de cruce (crossing parameters). Este modelo, no considerado por Marsden (1988), incorpora coeficientes multiplicativos que permiten que la interacción entre miembros de diferentes grupos sociales pueda tener niveles variables de dificultad, que además se acumulan entre categorías sociales (esto es, $v_{i j}=\sum_{u=i}^{i-1} v_{u}$ cuando $i \neq j) .{ }^{12}$ Estos niveles no asumen, como en el modelo de asociacion uniforme,

12. La expresión matemática de este modelo es más elaborada, pero Powers y Xie (2008: 117118) proveen una excelente revisión. 
un patrón lineal determinado por la distancia social. En cambio, el modelo permite capturar discontinuidades en los patrones de interacción intergrupal donde ciertas combinaciones de grupos sociales pueden ser marcadamente más o menos frecuentes que la interacción entre otras combinaciones. A modo de ejemplo, la estructura social de una sociedad podría favorecer la interacción entre personas con diferentes denominaciones religiosas, mientras que impide la interacción entre personas religiosas e irreligiosas. Estimamos este modelo bajo el supuesto de endogamia diferenciada.

A diferencia de Marsden (1988), no reportamos resultados de modelos log multiplicativos, ya que estos no agregan valor interpretativo adicional a los modelos ya descritos. Más aún, con la excepción de las tablas que cruzan la edad de los egos y alteri, los modelos multiplicativos con endogamia diferenciada consumen todos los grados de libertad disponibles, por lo que equivalen a un modelo saturado, y por ende no tiene ningún valor teórico.

\section{Resultados estadísticos}

Los resultados principales del análisis se muestran para cada dimensión en el cuadro 1. Este provee los estadísticos de ajuste comúnmente usados en la literatura de modelos log lineales, a saber, la Devianza $\left(\mathrm{G}^{2}\right)$, el Criterio de información bayesiano (BIC) y el índice de Disimilitud (D). ${ }^{13}$ Bajo la columna Gl se indican los Grados de libertad residuales asociados a cada modelo. Nuestra lectura de los resultados se concentra en el ajuste de los modelos en cada dimensión más que en revisar detalladamente los parámetros, dado que nuestro objetivo consiste en revelar la estructura general de la asociación entre encuestados y confidentes. Presentamos primero nuestro análisis dimensión por dimensión para el total de díadas observadas, y posteriormente consideramos las diferencias según el tipo de vínculo.

\section{Sexo}

Dado que esta variable solo adopta 2 valores, la resultante tabla de contingencia de $2 \times 2$ no permite estimar más que el modelo de independencia. Dados los valores muy elevados de la devianza y del índice de disimilitud, podemos concluir, sin embargo, que el modelo no se ajusta a los datos y, por tanto, el sexo de ego y alter inciden en la frecuencia de la interacción social. La asociación entre egos y alteri en esta dimensión puede expresarse en la razón de momios, que en este caso es de 3,95. Vale decir que las chances de encontrar una mujer entre los contactos de mujeres, comparadas con las chances de encontrar una mujer entre los contactos de hombres, son casi cuatro veces mayores. Lo anterior muestra claramente el sesgo de género en las redes de conversación.

13. El estadístico $D$ mide el porcentaje de casos que habría que mover entre las celdas de la tabla para obtener un ajuste perfecto. Formalmente corresponde a: $D=\left(\sum n_{i}-\hat{\mu}\right) / 2 n$, donde $\hat{\mu}$
indica el número predicho de casos en una celda de la tabla. 
Cuadro 1. Estadísticos de bondad de ajuste de modelos log lineales de díadas encuestadoconfidentes

\begin{tabular}{llrrrrc}
\hline Variable & Modelo & \multicolumn{1}{c}{$\mathrm{G}^{2}$} & $\mathrm{Gl}$ & \multicolumn{1}{c}{ BIC } & $\mathrm{D}$ & $\mathrm{N}$ \\
\hline Sexo & Independencia & 644,592 & 1 & 635,925 & 0,164 & 5.810 \\
\hline Edad & Independencia & $2.842,436$ & 25 & $2.627,050$ & 0,289 & 5.516 \\
& Endogamia constante (EC) & 696,847 & 24 & 490,077 & 0,127 & 5.516 \\
& Endogamia diferenciada (ED) & 400,188 & 19 & 236,495 & 0,082 & 5.516 \\
& Cuasisimetría & 40,581 & 10 & $-45,574$ & 0,027 & 5.516 \\
& Asociación uniforme con EC & 213,667 & 23 & 15,513 & 0,077 & 5.516 \\
& Asociación uniforme con ED & 129,126 & 18 & $-25,952$ & 0,046 & 5.516 \\
& Cruce de parámetros & 123,320 & 16 & $-14,526$ & 0,042 & 5.516 \\
\hline Educación & Independencia & $1.488,509$ & 9 & $1.411,804$ & 0,227 & 5.028 \\
& Endogamia constante (EC) & 448,401 & 8 & 380,219 & 0,105 & 5.028 \\
& Endogamia diferenciada (ED) & 341,831 & 5 & 299,217 & 0,077 & 5.028 \\
& Cuasisimetría & 4,900 & 3 & $-20,668$ & 0,007 & 5.028 \\
& Asociación uniforme con EC & 36,641 & 7 & $-23,019$ & 0,025 & 5.028 \\
& Asociación uniforme con ED & 13,290 & 4 & $-20,801$ & 0,010 & 5.028 \\
& Cruce de parámetros & 4,973 & 4 & $-29,119$ & 0,007 & 5.028 \\
\hline Religión & Independencia & $1.927,014$ & 9 & $1.850,041$ & 0,229 & 5.180 \\
& Endogamia constante (EC) & 167,740 & 8 & 99,319 & 0,055 & 5.180 \\
& Endogamia diferenciada (ED) & 9,584 & 5 & $-33,179$ & 0,006 & 5.180 \\
& Cuasisimetría & 5,697 & 3 & $-19,961$ & 0,006 & 5.180 \\
& Cruce de parámetros & 9,481 & 4 & $-24,729$ & 0,006 & 5.180 \\
\hline Posición & Independencia & $1.366,347$ & 9 & $1.288,339$ & 0,173 & 5.811 \\
política & Endogamia constante (EC) & 286,436 & 8 & 217,096 & 0,070 & 5.811 \\
& Endogamia diferenciada (ED) & 74,258 & 5 & 30,921 & 0,028 & 5.811 \\
& Cuasisimetría & 11,769 & 3 & $-14,234$ & 0,010 & 5.811 \\
& Cruce de parámetros & 11,921 & 4 & $-22,749$ & 0,010 & 5.811 \\
\hline
\end{tabular}

Fuente: elaboración propia según ENACOES 2014.

\section{Edad}

El modelo que obtiene el mejor ajuste es el de cuasisimetría $\left(\mathrm{G}^{2}=40,58\right.$; $\mathrm{BIC}=-45,57)$. Refleja alta propensión endogámica y que la interacción entre egos de mayor edad con alteri más jóvenes ocurre con una frecuencia similar a la entre egos jóvenes y alteri de mayor edad. Otros dos modelos, aunque ofrecen menor ajuste, permiten especificar más detalladamente la pauta de asociación: el modelo de parámetros de cruce $\left(\mathrm{G}^{2}=123,3\right.$; $\left.\mathrm{BIC}=-14,53\right)$ y el de asociación uniforme con homofilia diferenciada $\left(\mathrm{G}^{2}=129,13 ; \mathrm{BIC}=-25,95\right)$. Entre estos dos últimos, el modelo de asociación uniforme ofrece el mejor BIC, dado que se especifica usando solamente un parámetro y debería preferirse por su parsimonia. El parámetro de interacción entre la edad de los encuestados y los confidentes es positivo y significativo $(\beta \mathrm{EC}=0.15 ; \mathrm{p}<0.001))$. Esto implica que las chances de interacción entre un encuestado con un confidente del grupo etario siguiente 
(más viejo) son un $16 \%$ mayores que las chances de interacción con un confidente de un grupo etario contiguo menor.

\section{Educación}

Los modelos de cuasisimetría $\left(\mathrm{G}^{2}=4,9\right)$ y de parámetros de cruce con endogamia diferenciada $\left(\mathrm{G}^{2}=4,97\right)$ ofrecen el mejor ajuste a la pauta de relaciones entre encuestados y confidentes según el nivel educacional. Aunque otros modelos obtienen buenos estadísticos BIC, el de cruce de parámetros ofrece a la vez el mejor ajuste y el mejor BIC.

Los modelos de asociación uniforme, con endogamia constante o diferenciada, apuntan en la dirección de una continuidad subyacente a la pauta de relación cuando ego y alter poseen distintos niveles de educación. Ambos obtienen coeficientes interactivos positivos y altamente significativos $\left(\beta_{E C}=0,42\right.$; $p<0,001$ y $\beta_{E C}=0,49 ; p<0,001$, respectivamente). Esto implica, de acuerdo con el modelo con endogamia diferenciada, que las chances de interacción entre un encuestado y un confidente con un grado educacional más alto son un $63 \%$ mayores que las chances de interacción con un confidente de un grupo educacional contiguo menor.

El modelo de parámetros de cruce apunta a la existencia de barreras, bajo la forma de menores probabilidades de interacción entre algunas categorías contiguas. Sin constituir una discontinuidad infranqueable, algunos peldaños de las categorías educativas son más altos que otros. En particular, la interacción entre personas con educación media (secundaria) y superior técnica $\left(v_{2}=-0,88 ; p<0,001\right)$ son menos frecuentes que las relaciones entre personas con educación básica y secundaria $\left(v_{1}=-0,71 ; p<0,001\right.$, y entre aquellos con educación superior técnica y universitaria $\left(v_{3}=-0,53 ; p<0,001\right)$. De este modo, existe una suerte de división social entre las personas que alcanzaron y no alcanzaron la educación superior.

\section{Religión}

Las estimaciones para religión muestran cuatro modelos con muy baja devianza, liderados por el modelo de cuasisimetría $\left(\mathrm{G}^{2}=5,7\right)$, seguido por el de parámetros de cruce $\left(\mathrm{G}^{2}=9,48\right)$. No obstante, la especificación más parsimoniosa de acuerdo con el BIC corresponde al modelo de cuasiindependencia con homofilia diferenciada $(\mathrm{BIC}=-33,18)$. En términos del índice de disimilitud, este último modelo y los dos anteriores son semejantes y muestran valores extremadamente bajos $(\mathrm{D}=0,006)$. Vale decir que habría que mover solamente el $0,6 \%$ de las observaciones a distintas celdas de la tabla para obtener ajuste perfecto. Esto nos indica que la frecuencia de las relaciones sociales cercanas entre miembros de un mismo grupo religioso es muy preponderante, mientras que entre miembros de distintos grupos religiosos se acerca a la independencia estadística, es decir, que están determinadas mayormente por el tamaño de cada grupo religioso. 


\section{Politica}

Para las díadas según posición política, el modelo de parámetros de cruce $\left(\mathrm{G}^{2}=11,9 ; \mathrm{D}=0,01\right)$ y el de cuasisimetría ajustan adecuadamente los datos $\left(\mathrm{G}^{2}=11,77 ; \mathrm{D}=0,01\right)$. La cuasisimetría indica que la interacción cercana entre egos y alteri de diferentes convicciones políticas tiende a ser recíproca, de modo que no hay un grupo político que tienda a rechazar de manera unilateral a miembros de otros grupos. El modelo de cruce de parámetros, por su parte, precisa que existen algunas categorías políticas en las cuales resulta menos probable que se produzca la comunicación. En particular, los coeficientes que capturan la frecuencia de interacción cercana entre los independientes y aquellos identificados políticamente, sea de la tendencia que sea, son todos negativos y estadísticamente significativos. ${ }^{14}$

Para expresar nuestros resultados de un modo más intuitivo, comparamos en el gráfico 2 los niveles estimados de endogamia grupal sugeridos por el modelo que obtiene el menor BIC y el modelo de independencia. Como indica el gráfico, las relaciones entre personas de similar edad, igual nivel educacional, posición política y religión son marcadamente más frecuentes que lo esperado bajo el supuesto de independencia, aunque ciertamente hay patrones variables. Por ejemplo, del total de díadas entre encuestados y confidentes observadas entre los encuestados de 18 a 24 años, el modelo de cuasisimetría predice que un $65 \%$ de esas relaciones se establecen con alteri del mismo grupo etario, en tanto el modelo de independencia predice solo un $19 \%$. Esto implica que en Chile las relaciones entre personas de 18 a 24 años son 3,4 veces más frecuentes de lo que lo serían si las relaciones sociales se rigieran aleatoriamente según el tamaño de los grupos. Entre los grupos etarios de 24-34 y 55-65, los niveles de endogamia se reducen algo, pero esta propensión nunca deja de ser cercana al doble de lo frecuente que sería bajo el supuesto de independencia. En contraste, en el grupo etario mayor, la endogamia se incrementa fuertemente, donde las relaciones de mayores de 65 años son 4,4 veces más frecuentes de lo que se espera bajo el supuesto de independencia.

Los niveles de endogamia grupal según el nivel educacional de las personas son relativamente altos, salvo en el caso de las personas con educación media (completa o incompleta). Entre los encuestados con educación básica o menos, el modelo de cruce de parámetros predice que un $34 \%$ de sus relaciones se establecen con confidentes del mismo nivel, mientras que el modelo de independencia predice solo un $12 \%$, esto es 2,8 veces más de lo esperado bajo el supuesto de

14. Los coeficientes que capturan la frecuencia de interacción, en escala logarítmica, entre personas de derecha y centro (DC), centro e izquierda (CI), e izquierda e independientes (II) son: $v_{D C}=-0,60, p<0,001 ; v_{C I}=0,39, p<0,001$ y $v_{I I}=-1,18, p<0,001$. En consecuencia, el coeficiente que captura la frecuencia de conversación entre personas de derecha e independientes equivale a: $v_{D I}=-0,6+0,39-1,18=-1,39$, y entre personas de centro e independientes es: $v_{C I}=0,39-1,18=-0,79$. En otras palabras, todos los coeficientes son negativos, lo que indica que la frecuencia de conversación entre personas con estas inclinaciones políticas es menos frecuente. 
Gráfico 2. Proporción estimada de díadas encuestados-confidentes según edad, educación, posición política y religión de los encuestados según el modelo con mejor BIC y el modelo de independencia.

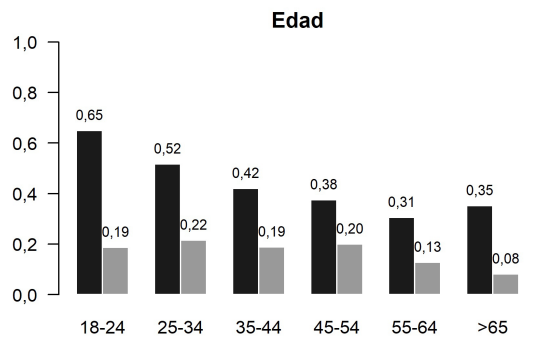

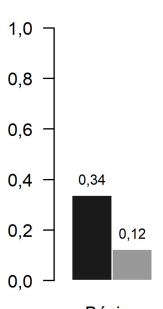

Básica
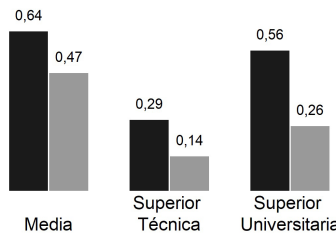

Religión
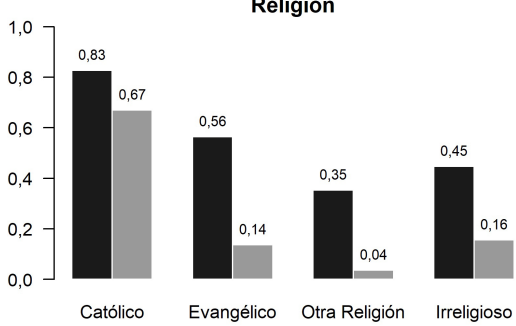

Modelo de Independencia

Fuente: elaboración propia según ENACOES 2014.

independencia. Entre los encuestados con educación superior técnica y universitaria (completa o incompleta), esta razón es igual a 2,1 y 2,2 veces más alta. En cambio, entre las personas con educación media, es solo 1,4 veces mayor.

Cuando consideramos la posición política y la religión de los entrevistados, encontramos mayores niveles de variación intergrupal. Las personas que no se identifican con ninguna posición política registran un nivel absoluto excepcionalmente alto de lazos intragrupales, pero si se contrasta con el modelo de independencia la razón es solo 1,4 veces mayor que si las relaciones dependieran exclusivamente del tamaño de los grupos políticos. En cambio, las personas de izquierda y derecha tienden a relacionarse con alteri ideológicamente parecidos en mayor medida, a saber, 2,2 y 3,4 veces más, respectivamente. De forma similar, el grupo religioso más voluminoso, los católicos, tiende a relacionarse más con otros católicos, pero, en contraste con el modelo de independencia, lo hace solo 1,2 veces más. En cambio, la frecuencia de las relaciones entre los demás grupos religiosos es marcadamente más alta que las sugeridas por el modelo de independencia: 4 veces para los evangélicos, 8,8 veces para miembros de religiones minoritarias, y 2,8 veces para la población irreligiosa.

Finalmente, consideramos los resultados diferenciando si las relaciones entre encuestados y confidentes son de tipo familiar (esposo/a, hermanos, 
Gráfico 3. Comparación niveles de endogamia entre díadas de encuestados y confidentes según tipo de relación. Todas las proporciones han sido calculadas según los M2 del cuadro $2 \mathrm{D}$.
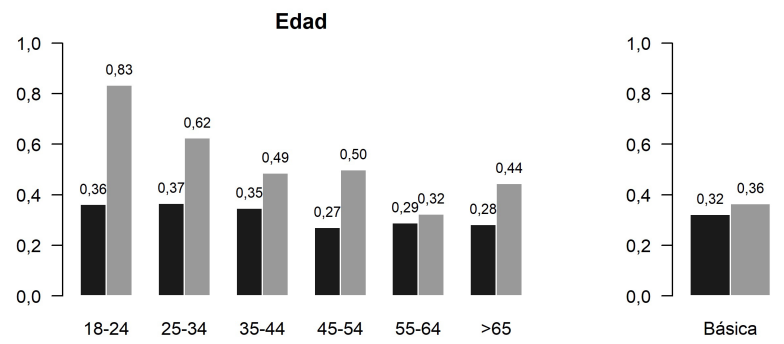

Educación

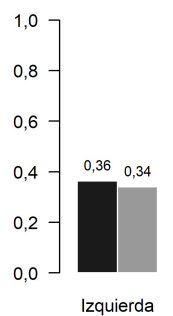

Posición Política
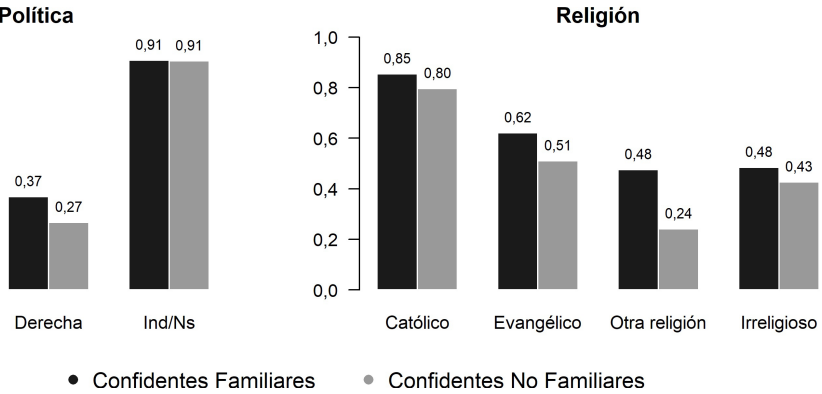

- Confidentes Familiares

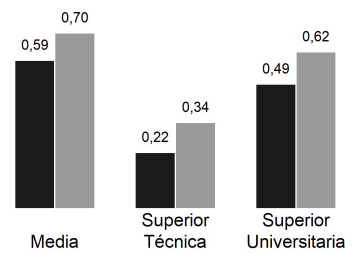

Fuente: elaboración propia según ENACOES 2014.

padres y otros) o no familiar (amigos, vecinos, colegas y otros). Los resultados se detallan en los cuadros $1 \mathrm{C}$ y $2 \mathrm{C}$ de la sección $\mathrm{C}$ del apéndice. De este análisis se desprenden dos resultados relevantes. Primero, los modelos con mayor capacidad de ajuste para el total de la muestra de díadas tienden a ser los mismos para el subconjunto de díadas familiares y no familiares. En consecuencia, el modelo de cuasisimetría y el de cruce de parámetros obtienen buen ajuste para todas la variables de homofilia, tanto para díadas familiares como no familiares. Para el caso de religión, el modelo de cuasiindependencia obtiene estadísticos BIC marginalmente superiores a los dos modelos anteriores.

En segundo lugar, estimamos un conjunto de modelos de cuasisimetría de tres dimensiones para todas las variables de homofilia, donde cruzamos la membrecía grupal del encuestado, confidente y el tipo de relación (familiar o no familiar). Se estiman dos tipos de modelos. En el primero, solo se controla por el tipo de relación entre los encuestados y confidentes, mientras que el segundo multiplica esta variable con todas las variables que capturan interacciones intra e intergrupales. Sin excepción alguna, los resultados favorecen esta última especificación y, por ende, indican que los niveles de endogamia varían significativamente según el tipo de relación. En el gráfico 3, presentamos los niveles de endogamia grupal para cada variable según este último modelo. Se 
puede notar que la endogamia etaria y educacional es más alta entre confidentes no familiares, algo observado por Marsden (1988) en Estados Unidos, mientras que la endogamia política y religiosa se ve reforzada entre confidentes familiares. No obstante, la magnitud de las diferencias es realmente considerable solamente para la edad y, en menor grado, para el nivel educacional. El contraste etario más fuente se encuentra entre los encuestados más jóvenes de la muestra. Mientras que el $36 \%$ de las relaciones familiares ocurre con alteri del mismo grupo etario, este número alcanza el $83 \%$ entre relaciones no familiares. Esta brecha tiende a reducirse parcialmente hasta el penúltimo grupo etario, ya que vuelve a crecer entre las personas mayores de 65 años.

Los grupos educacionales también muestran algunas diferencias de importancia, particularmente entre las díadas con nivel educacional medio (secundaria) y universitario, donde la endogamia es 13 puntos porcentuales más alta entre relaciones no familiares que familiares. También destacan los grupos religiosos minoritarios (otra religión), donde la endogamia es 24 puntos porcentuales más alta entre familiares que entre no familiares, algo que no se reproduce en los demás grupos religiosos.

\section{Discusión de resultados y conclusiones}

Nuestros resultados indican cuatro pautas comunes a las dimensiones de homofilia en la sociedad chilena. En primer lugar, y descontando la disponibilidad demográfica de las distintas categorías sociales, las relaciones sociales son marcadamente más frecuentes entre personas socialmente similares, y particularmente cuando comparten igual membrecía grupal. Esto no solo confirma la hipótesis 1 respecto al peso de las relaciones intragrupales, sino que se alinea con la investigación previa sobre homofilia hecha en Europa y Norteamérica. Los atributos que tienden a generar mayores niveles de interacción intragrupal son la edad y la religión $(2,7$ y 2,4 veces más frecuentes, respectivamente), seguidas por la posición política y el nivel educacional (ambos 1,8 veces más frecuentes). En términos globales, las relaciones sociales endogámicas son, en promedio, 2,2 veces más frecuentes de lo que serían si la misma membrecía grupal no influyera sobre la formación de lazos sociales. ${ }^{15}$ En terminología de Blau, las variables de diferenciación consideradas en el análisis constituyen efectivamente parámetros estructurales. Estos resultados también contrastan con estudios previos que ponen mucho énfasis en el elevado nivel de segregación educacional del sistema educativo chileno (Valenzuela et al., 2014). Nuestras estimaciones no indican lo contrario, pero sí ponen de manifiesto que hay otras dimensiones sociales donde la frecuencia de las relaciones cercanas es aún más endogrupal.

Segundo, todos los modelos que estiman homofilia diferenciada ajustan mejor los datos que aquellos con homofilia constante y, consecuentemente, indican

15. Calculamos este promedio ponderando por el tamaño de cada grupo social, el que obtenemos a partir de la distribución marginal de cada variable de homofilia reportada por los encuestados. 
que los niveles de interacción social intragrupal varían significativamente entre las distintas categorías sociales. En el caso de la posición política y la religión, es mucho menor entre los grupos mayoritarios que entre los grupos minoritarios, y en el caso de la edad y el nivel educacional se concentra mayormente en los grupos localizados en las puntas (jóvenes y adultos mayores, y educación básica y universitaria). De esta forma, las estimaciones apuntan a que en la sociedad chilena la "clausura del grupo» es una característica dominante, pero sujeta a variación según la categoría social considerada. Más aún, los patrones de homofilia informan acerca de posibles amenazas a cohesión social global de la sociedad chilena, donde la interacción entre miembros de ciertos grupos sociales es muy reducida.

Tercero, y a pesar de las generalizaciones anteriores, diversos aspectos de la homofilia encuentran sustento en los múltiples modelos estimados. Varios modelos ofrecen un ajuste razonable de los datos $y$, por ende, no hay un sustento estadístico irrefutable para optar por uno u otro, mientras que la interpretación sustantiva y la parsimonia de los modelos pueden llevar a preferir otros. El modelo de cuasisimetría, que obtiene una elevada capacidad predictiva para todas las dimensiones de la homofilia, habla a favor de la preferencia de los chilenos a establecer relaciones cercanas con otros similares, y de la ausencia de favoritismos grupales unilaterales. El modelo de semiindependencia ajusta muy bien para la religión, e indica fuerte endogamia intragrupal, mientras que la interacción interreligiosa está determinada mayormente por disponibilidad demográfica. El modelo de asociación uniforme ajusta adecuadamente para edad, e indica que las chances de encuentro entre personas con diferencias etarias tienden a reducirse linealmente. Por otro lado, los modelos de parámetros de cruce indicarían que existen barreras de contacto en las dimensiones de educación y de posición política. En el primer caso, los modelos ponen de manifiesto una suerte de clivaje que divide a las personas que tienen o no tienen educación superior, mientras que, en el segundo, hay una marcada discontinuidad en el nivel de interacción entre personas con y sin preferencias ideológicas definidas. Esto da respaldo a la hipótesis 2, que indicaba que la frecuencia de las relaciones sociales entre miembros de distintos grupos etarios y educacionales se reducirá a medida que la distancia social (etaria o educacional) se acreciente también. Para el caso de educación habría que agregar que la distancia social no opera de forma enteramente lineal, dada la existencia de las barreras detectadas.

Cuarto, hay marcadas similitudes en el ajuste de los modelos para familiares y para no familiares. Es decir, las pautas que caracterizan la homofilia en las relaciones de amistad también lo hacen para las relaciones entre familiares. No obstante, los datos no permiten establecer la dirección en la cual opera el efecto, esto es, si las relaciones de confianza se asimilan con las familiares o bien constituyen una pauta general que no depende del parentesco.

Junto con proveer una caracterización multidimensional de la propensión a la homofilia en Chile, nuestro estudio abre nuevos interrogantes y líneas de investigación. Dada la alta prevalencia de relaciones homofilicas, creemos que una mayor investigación sobre este tema es primordial. Aquí sugerimos algunas posibilidades. 
En primer lugar, y siguiendo lo propuesto por Blau (1977), sería necesario estudiar cómo distintos grupos sociales se van consolidando en la medida en que distintos parámetros sociales se van correlacionando. En otras palabras, la homofilia en una dimensión podría potenciar mayor homofilia en otras dimensiones. Por ejemplo, las relaciones homofílicas entre personas con bajo nivel educacional podrían fomentar, indirectamente, las relaciones homofílicas entre personas con edades similares. Este aspecto tiene una especial importancia para la cohesión social, pues tener relaciones predominantemente homofílicas en varias dimensiones simultáneamente implica observar un limitado horizonte social de la realidad, lo que juega un rol clave en la reproducción de las diferencias económicas y culturales de diferentes grupos demográficos (DiMaggio y Garip, 2011).

En segundo lugar, es importante conocer qué factores sociales contextuales promueven mayores niveles de homofilia. A modo de ejemplo, el aumento de la desigualdad económica, expresado en factores como los niveles de segregación residencial - fenómeno de alta prevalencia en las grandes metrópolis latinoamericanas - o la segregación socioeconómica de los sistemas educacionales, podrían, presumiblemente, reducir la propensión que personas socialmente disimilares pueden interactuar de forma recurrente.

En tercer lugar, hasta la fecha la gran mayoría de los estudios sobre homofilia social han sido de corte transversal. La única excepción importante realizada en el contexto de Estados Unidos es el sofisticado estudio de Smith et al. (2014), que investiga los niveles de homofilia en dos puntos en el tiempo. Más interesante aún sería poder estudiar, sobre la base de estudios de panel, los niveles de variación o estabilidad de la homofilia para un mismo grupo de individuos. Esto permitiría analizar de qué manera circunstancias ocurridas durante el ciclo de vida de las personas, como por ejemplo el ingreso o la retirada del mercado laboral, o cambios en los niveles de involucramiento religioso o político, podrían incidir en los niveles de homofilia de las relaciones sociales.

\section{Financiamiento}

Los autores agradecen a ANID Chile su apoyo a través del Centro de Estudios de Conflicto y Cohesión Social (COES, fondo ANID/FONDAP/15130009), y del Concurso Fondecyt Regular 1171426 (IR Vicente Espinoza).

\section{Referencias bibliográficas}

Abascal, Maria y Baldassarri, Delia (2015). «Love Thy Neighbor? Ethnoracial Diversity and Trust Reexamined». American Journal of Sociology, 121 (3), 722-82. <https://doi.org/10.1086/683144>

Blau, Peter M. (1974). «Presidential Address: Parameters of Social Structure». American Sociological Review, 39 (5), 615-635. $<$ https://doi.org/10.2307/2094309>

- (1977). "A Macrosociological Theory of Social Structure». American Journal of Sociology, 83 (1), 26-54. <https://doi.org/10.1086/226505> 
Blau, Peter M.; Beeker, Carolyn y Fitzpatrick, Kevin M. (1984). «Intersecting Social Affiliations and Intermarriage». Social Forces, 62 (3), 585-606. <https://doi.org/10.1093/sf/62.3.585>

Blau, Peter M. y Schwartz, Joseph (1984). Cross-Cutting Social Circles: Testing a Macrosociological Theory of Intregroup Relations. Nueva York: Academic Press.

Brechwald, Whitney A. y Prinstein, Mitchell J. (2011). «Beyond Homophily: A Decade of Advances in Understanding Peer Influence Processes». Journal of Research on Adolescence, 21 (1), 166-79. <https://doi.org/10.1111/j.1532-7795.2010.00721.x>

Burt, Ronald S. (1984). "Network Items and the General Social Survey». Social Networks, 6 (4), 293-339. <https://doi.org/10.1016/0378-8733(84)90007-8>

DiMaggio, Paul y Garip, Filiz (2011). «How Network Externalities Can Exacerbate Intergroup Inequality». American Journal of Sociology, 116 (6), 1.887-1.933. <https://doi.org/10.1086/659653>

Espinoza, Vicente y Canteros, Eduardo (2001). «Contactos sociales y carreras laborales en hogares chilenos de escasos recursos». Proposiciones, 32, 170-89.

Ferrand, Alexis; Mounier, Lise y Degenne, Alain (1999). «The Diversity of Personal Networks in France: Social Stratification and Relational Structures». En: WelLman, Barry (eds.). Networks in the Global Village. Life in Contemporary Communities. Nueva York: Routledge.

FIsCher, Claude S. (2009). "The 2004 GSS Finding of Shrunken Social Networks: An Artifact?». American Sociological Review, 74 (4), 657-69. <https://doi.org/10.1177/000312240907400408>

Forsé, Michel y Chauvel, Louis (1995). «L'évolution de l'homogamie en France. Une méthode pour comparer les diagonalités de plusieurs tables». Revue Française de Sociologie, 36 (1), 123-42. <https://doi.org/10.2307/3322313>

Granovetter, Mark S. (1973). «The Strength of Weak Ties». American Journal of Sociology, 78 (6), 1.360-80. <https://doi.org/10.1086/225469>

Gullickson, Aaron y Torche, Florencia (2014). «Patterns of Racial and Educational Assortative Mating in Brazil». Demography, 51 (3), 835-856. <https://doi.org/10.1007/s13524-014-0300-2>

Hampton, Keith N. (2016). «Persistent and Pervasive Community: New Communication Technologies and the Future of Community». American Behavioral Scientist, 60 (1), 101-24. <https://doi.org/10.1177/0002764215601714>

Hampton, Keith N.; Sessions, Lauren F. y Ja Her, Eun (2011). "Core Networks, Social Isolation, and New Media: How Internet and Mobile Phone Use Is Related to Network Size and Diversity». Information, Communication \& Society, 14 (1), 130-55. <https://doi.org/10.1080/1369118X.2010.513417>

Hoffman, Kelly y Centeno, Miguel (2003). «The Lopsided Continent: Inequality in Latin America». Annual Review of Sociology, 29 (1), 363-390. <https://doi.org/10.1146/annurev.soc.29.010202.100141>

Kalmijn, Matthijs (1998). "Intermarriage and Homogamy: Causes, Patterns, Trends». Annual Review of Sociology, 24 (1), 395-421. <https://doi.org/10.1146/annurev.soc.24.1.395> 
Laumann, Edward O. y Senter, Richard (1976). «Subjective Social Distance, Occupational Stratification, and Forms of Status and Class Consciousness: A Cross-National Replication and Extension». American Journal of Sociology, 81 (6), 1.304-1.338. <https://doi.org/10.1086/226225>

LaZArsfeld, Paul F. y Merton, Robert K. (1954). «Friendship as a Social Process: A Substantive and Methodological Analysis». En: Berger, Morroe; Abel, Theodore y Page, Charles H. (eds.). Freedom and Control in Modern Society. Nueva York: Van Nostrand.

Louch, Hugh (2000). «Personal Network Integration: Transitivity and Homophily in Strong-Tie Relations». Social Networks, 22, 45-64. <https://doi.org/10.1016/S0378-8733(00)00015-0>

Lozares, Carlos y Verd, Joan Miquel (2011). «De la homofilia a la cohesión social y viceversa». Redes. Revista Hispana para el Análisis de Redes Sociales, 20 (1), 29-50. <https://doi.org/10.5565/rev/redes.408>

Lozares, Carlos; Verd, Joan Miquel; Cruz, Irene y Barranco, Oriol (2014). «Homophily and Heterophily in Personal Networks. From Mutual Acquaintance to Relationship Intensity». Quality \& Quantity, 48 (5), 2.657-70. $<$ https://doi.org/10.1007/s11135-013-9915-4>

Lumley, Thomas (2017). Survey: Analysis of Complex Survey Samples. R Package Version 3.32 .

Luna, Juan Pablo y Altman, David (2011). «Uprooted but Stable: Chilean Parties and the Concept of Party System Institutionalization». Latin American Politics and Society, 53 (2), 1-28. <https://doi.org/10.1111/j.1548-2456.2011.00115.x>

Marin, Alexandra (2004). "Are Respondents More Likely to List Alters with Certain Characteristics?». Social Networks, 26 (4), 289-307. <https://doi.org/10.1016/j.socnet.2004.06.001>

Marsden, Peter V. (1987). "Core Discussion Networks of Americans». American Sociological Review, 52 (1), 122-131. <https://doi.org/10.2307/2095397>

- (1988). «Homogeneity in Confiding Relations». Social Networks, 10 (1), 57-76. <https://doi.org/10.1016/0378-8733(88)90010-X>

- (1990). «Network Data and Measurement». Annual Review of Sociology, 16, 435-63. <https://doi.org/10.1146/annurev.so.16.080190.002251>

McPherson, Miller; Smith-Lovin, Lynn y Cook, James M. (2001). "Birds of a Feather: Homophily in Social Networks». Annual Review of Sociology, 27 (1), 415-444. <https://doi.org/10.1146/annurev.soc.27.1.415>

McPherson, Miller; Smith-Lovin, Lynn y Brashears, Matthew E. (2006). «Social Isolation in America: Changes in Core Discussion Networks over Two Decades». American Sociological Review, 71 (3), 353-375. <https://doi.org/10.1177/000312240607100301>

McPherson, Miller; Smith-Lovin, Lynn y Brashears, Matthew E. (2009). «Models and Marginals: Using Survey Evidence to Study Social Networks». American Sociological Review, 74 (4), 670-681. <https://doi.org/10.1177/000312240907400409>

Miguel Luken, Verónica de y Tranmer, Mark (2010). «Personal Support Networks of Immigrants to Spain: A Multilevel Analysis». Social Networks, 32 (4), 253-262. <https://doi.org/10.1016/j.socnet.2010.03.002> 
Mollenhorst, Gerald; Beate, Volker y Henk, Flap (2008). «Social Contexts and Core Discussion Networks: Using a Choice-Constraint Approach to Study Similarity in Intimate Relationships». Social Forces, 86 (3), 937-65. <https://doi.org/10.1353/sof.0.0010>

Powers, Daniel y Xie, Yu (2008). Statistical Methods for Categorical Data Analysis. Emerald Group Publishing.

Ramos-Vidal, Ignacio (2016). "Popularidad y relaciones entre iguales en el aula: un estudio prospectivo». Psicología Educativa, 22 (2), 113-24. <https://doi.org/10.1016/j.pse.2015.12.001>

Ramos-Vidal, Ignacio; Castro, Belkis y Palacio, Jorge (2016). «Patrones de interacción y grupos de discusión política en las redes personales de jóvenes colombianos». América Latina Hoy, 73 (0), 121. <https://doi.org/10.14201/alh201673121139>

Ramos-Vidal, Ignacio; Villamil, Ilse y Uribe, Alicia (2019). "Underlying Dimensions of Social Cohesion in a Rural Community Affected by Wartime Violence in Colombia». International Journal of Environmental Research and Public Health, $16(2), 195$. <https://doi.org/10.3390/ijerph16020195>

Rodríguez, Santiago A. (2016). "Selección de parejas y estratificación social: hacia una agenda de investigación». Estudios Sociológicos, 34 (100), 169-190. <https://doi.org/10.24201/ES.2016V34N100.1400>

Silva, Patricio (2004). «Doing Politics in a Depoliticised Society: Social Change and Political Deactivation in Chile». Bulletin of Latin American Research, 23 (1), 63-78. <https://doi.org/10.1111/j.1470-9856.2004.00096.x>

Smith, Jeffrey A.; McPherson, Miller y Smith-Lovin, Lynn (2014). «Social Distance in the United States: Sex, Race, Religion, Age, and Education Homophily among Confidants, 1985 to 2004». American Sociological Review, 79 (3), 432-456. <https://doi.org/10.1177/0003122414531776>

Solís, Patricio (2010). «Entre un buen partido y un peor es nada: selección de parejas en la Ciudad de México». Revista Latinoamericana de Población, 4 (7), 57-78.

Torche, Florencia (2010). «Educational Assortative Mating and Economic Inequality: A Comparative Analysis of Three Latin American Countries». Demography, 47 (2), 481-502. <https://doi.org/10.1353/dem.0.0109>

Torche, Florencia y Valenzuela, Eduardo (2011). «Trust and Reciprocity: A Theoretical Distinction of the Sources of Social Capital». European Journal of Social Theory, 14 (2), 181-198. <https://doi.org/10.1177/1368431011403461>

Valenzuela, Eduardo y Ayala, Cristián (2011). «Homofilia, selección e influencia en un estudio longitudinal de drogas en población escolar». Psykhe, 20 (2), 101-14. <https://doi.org/10.4067/S0718-22282011000200009>

Valenzuela, Juan Pablo; Bellei, Cristian y Ríos, Danae de los (2014). «Socioeconomic School Segregation in a Market-Oriented Educational System. The Case of Chile». Journal of Education Policy, 29 (2), 217-241. <https://doi.org/10.1080/02680939.2013.806995>

Wright, Erik Olin y Cho, Donmoon (1992). "The Relative Permeability of Class Boundaries to Cross-Class Friendships: A Comparative Study of the United States, Canada, Sweden, and Norway». American Sociological Review, 57 (1), 85-102. $<$ https://doi.org/10.2307/2096146> 


\section{Apéndice}

\section{Resultados descriptivos}

Cuadro 1A. Estadísticos descriptivos variables de homofilia (porcentajes ponderados)

\begin{tabular}{llrrrr}
\hline & & $\begin{array}{c}\text { Casos } \\
\text { encuestados }\end{array}$ & $\begin{array}{c}\text { Proporción } \\
\text { encuestados }\end{array}$ & $\begin{array}{c}\text { Casos } \\
\text { confidentes }\end{array}$ & $\begin{array}{c}\text { Proporción } \\
\text { confidentes }\end{array}$ \\
\hline Sexo & Mujer & 1.041 & 0,55 & 3.060 & 0,53 \\
& Hombre & 841 & 0,45 & 2.750 & 0,47 \\
\hline Grupos & $18-24$ & 257 & 0,13 & 1.029 & 0,19 \\
Etarios & $25-34$ & 381 & 0,19 & 1.187 & 0,22 \\
& $35-44$ & 335 & 0,17 & 1.042 & 0,19 \\
& $45-54$ & 405 & 0,20 & 1.102 & 0,20 \\
& 55-64 & 359 & 0,18 & 704 & 0,13 \\
& $>65$ & 289 & 0,14 & 452 & 0,08 \\
\hline Nivel & Ed. básica & 372 & 0,19 & 626 & 0,12 \\
educacional & Ed. media (secundaria) & 865 & 0,43 & 2.370 & 0,47 \\
& Ed. superior técnica & 327 & 0,16 & 716 & 0,14 \\
& Ed. superior universitaria & 443 & 0,22 & 1.316 & 0,26 \\
\hline Religión & Católico & 1.240 & 0,61 & 3.470 & 0,67 \\
& Evangélico & 304 & 0,15 & 709 & 0,14 \\
& Otra religión & 89 & 0,04 & 190 & 0,04 \\
& Irreligioso & 393 & 0,19 & 811 & 0,16 \\
\hline Posición & Izquierda & 447 & 0,22 & 939 & 0,16 \\
política & Centro & 687 & 0,34 & 661 & 0,11 \\
& Derecha & 240 & 0,12 & 526 & 0,09 \\
& Independiente o No sabe & 650 & 0,32 & 3.685 & 0,63 \\
\hline
\end{tabular}

Fuente: elaboración propia según ENACOES 2014. 
Cuadro 2A. Distribución bivariada de díadas encuestados-confidentes (datos ponderados)

\begin{tabular}{|c|c|c|c|c|c|c|}
\hline \multirow[b]{2}{*}{ Ego } & \multicolumn{6}{|c|}{ Alter } \\
\hline & Mujer & Hombre & & & & \\
\hline Mujer & 0,68 & 0,32 & & & & \\
\hline \multirow[t]{2}{*}{ Hombre } & 0,35 & 0,65 & & & & \\
\hline & $18-24$ & $25-34$ & $35-44$ & $45-54$ & $55-64$ & $>65$ \\
\hline $18-24$ & 0,65 & 0,11 & 0,08 & 0,13 & 0,03 & 0,01 \\
\hline $25-34$ & 0,14 & 0,52 & 0,17 & 0,11 & 0,05 & 0,01 \\
\hline $35-44$ & 0,12 & 0,21 & 0,42 & 0,11 & 0,09 & 0,05 \\
\hline $45-54$ & 0,13 & 0,13 & 0,17 & 0,38 & 0,13 & 0,07 \\
\hline $55-64$ & 0,06 & 0,13 & 0,14 & 0,29 & 0,31 & 0,08 \\
\hline \multirow[t]{2}{*}{$\geq 65$} & 0,04 & 0,10 & 0,14 & 0,17 & 0,2 & 0,35 \\
\hline & Básica & Media & Superior técnica & Superior univ. & & \\
\hline Básica & 0,34 & 0,55 & 0,05 & 0,07 & & \\
\hline Media (secundaria) & 0,14 & 0,64 & 0,10 & 0,13 & & \\
\hline Superior técnica & 0,07 & 0,36 & 0,29 & 0,28 & & \\
\hline \multirow[t]{2}{*}{ Superior univ. } & 0,04 & 0,24 & 0,16 & 0,56 & & \\
\hline & Católico & Evangélico & Otra religión & Irreligioso & & \\
\hline Católico & 0,83 & 0,06 & 0,02 & 0,1 & & \\
\hline Evangélico & 0,34 & 0,56 & 0,01 & 0,09 & & \\
\hline Otra religión & 0,45 & 0,07 & 0,35 & 0,12 & & \\
\hline \multirow[t]{2}{*}{ Irreligioso } & 0,45 & 0,08 & 0,02 & 0,45 & & \\
\hline & Izquierda & Centro & Derecha & Ind/Ns & & \\
\hline Izquierda & 0,35 & 0,11 & 0,07 & 0,47 & & \\
\hline Centro & 0,13 & 0,16 & 0,09 & 0,62 & & \\
\hline Derecha & 0,17 & 0,15 & 0,31 & 0,36 & & \\
\hline Independiente/Ns & 0,05 & 0,03 & 0,01 & 0,91 & & \\
\hline
\end{tabular}

Nota: porcentajes calculados según el total de casos de cada fila.

Fuente: elaboración propia según ENACOES 2014. 


\section{B. Modelos multinivel multinomiales bayesianos de homofilia con y sin control} de tamaño de red cercana

Coeficientes de Nivel Educativo de Ego Prediciendo Nivel Educativo de Alteri

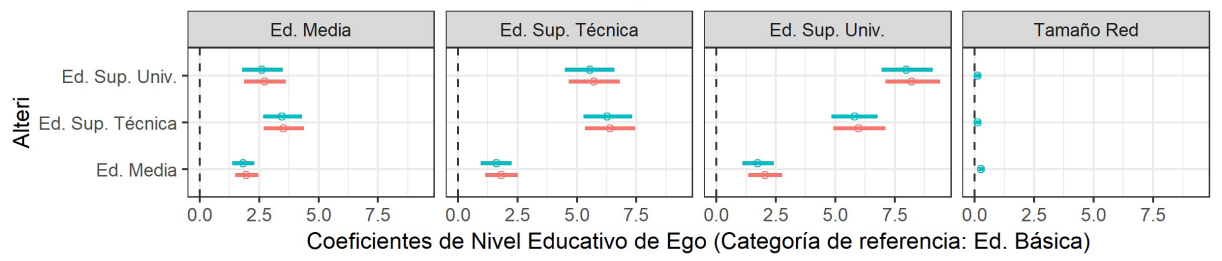

Coeficientes de Grupo Etario de Ego Prediciendo Grupo Etario de Alteri

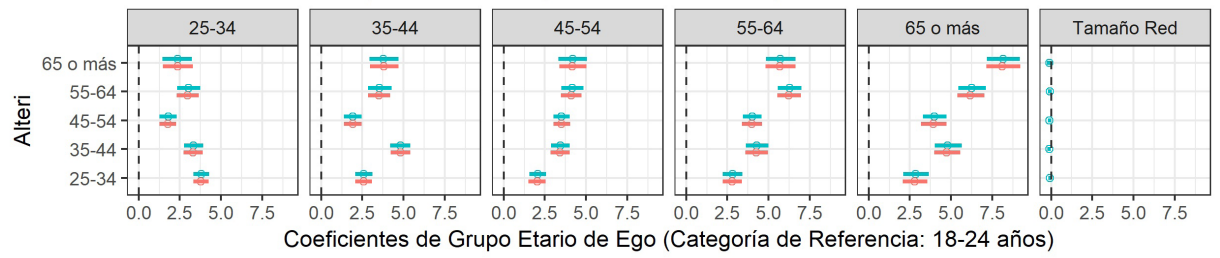

Coeficientes de Membecía Política de Ego Prediciendo Membrecía Política de Alteri

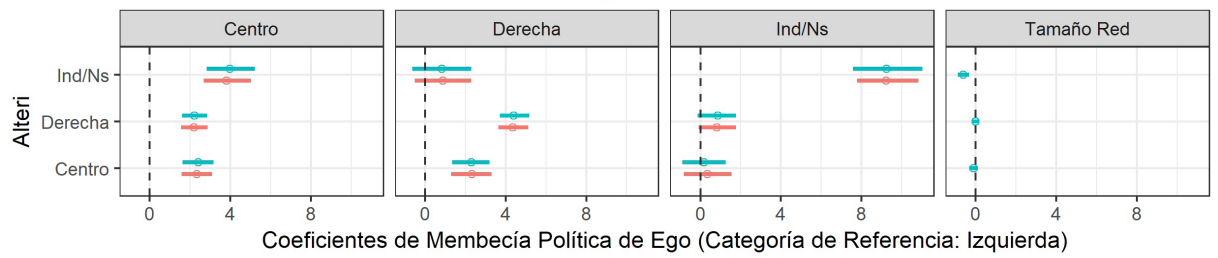

Coeficientes de Membecía Religiosa de Ego Prediciendo Membrecía Religiosa de Alteri

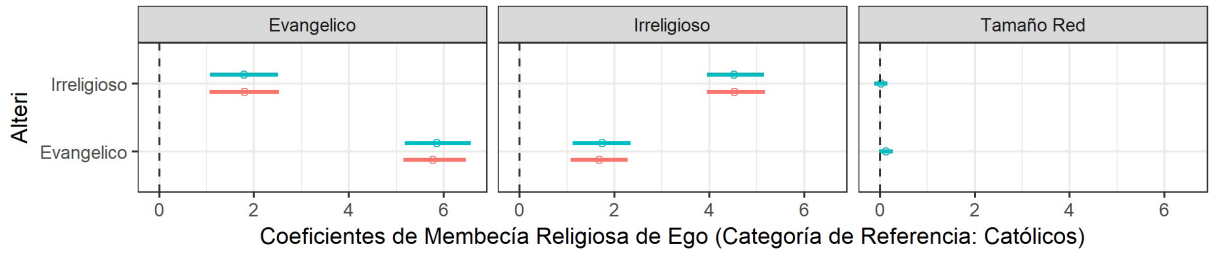

M1: sin control de $\&$ M2: con control de

Tamaño Red Tamaño Red

Nota: Cada gráfico representa los coeficientes de un modelo de regresión multinomial donde la membrecía grupal de los alteri (variable dependiente nominal) es función de la membrecía grupal de ego (variable independiente). Los segmentos horizontales corresponden a intervalos de credibilidad del $95 \%$ asociados a cada coeficiente. Los parámetros de cada modelo son estimados según tres cadenas de Markov basadas en 120.000 iteraciones, con las primeras 20.000 eliminadas (burn-in). Todos los coeficientes obtienen valores inferiores a 1,1 en la prueba de convergencia de Gelman y Rubin. 


\section{Resultados complementarios de modelos log lineales}

Cuadro 1C. Modelos log lineales de homofilia para relaciones familiares y no familiares

\begin{tabular}{|c|c|c|c|c|c|c|c|c|c|c|c|}
\hline & \multirow[b]{2}{*}{ Modelo } & \multicolumn{5}{|c|}{ Confidentes no familiares } & \multicolumn{5}{|c|}{ Confidentes familiares } \\
\hline & & $G^{2}$ & GI & BIC & D & $\mathrm{N}$ & $G^{2}$ & Gl & BIC & D & $\mathrm{N}$ \\
\hline Sexo & Independencia & 362,4871 & 1 & 354,4768 & 0,1715 & 3.012 & 274,5339 & 1 & 266,6314 & 0,1559 & 2.704 \\
\hline \multirow[t]{7}{*}{ Edad } & Independencia & $3.058,1627$ & 25 & $2.859,7105$ & 0,4314 & 2.802 & 565,381 & 25 & 368,484 & 0,175 & 2.633 \\
\hline & Endogamia constante (EC) & $1.039,9324$ & 24 & 849,4183 & 0,2165 & 2.802 & 227,239 & 24 & 38,2179 & 0,1021 & 2.633 \\
\hline & Endogamia diferenciada (ED) & 662,6428 & 19 & 511,8192 & 0,1385 & 2.802 & 154,791 & 19 & 5,1493 & 0,0769 & 2.633 \\
\hline & Cuasisimetría & 25,293 & 10 & $-54,0879$ & 0,0201 & 2.802 & 28,1439 & 10 & $-50,6149$ & 0,0358 & 2.633 \\
\hline & Asociación uniforme con EC & 276,8441 & 23 & 94,268 & 0,1152 & 2.802 & 179,9076 & 23 & $-1,2376$ & 0,1019 & 2.633 \\
\hline & Asociación uniforme con ED & 127,3454 & 18 & $-15,5402$ & 0,0523 & 2.802 & 135,3566 & 18 & $-6,4093$ & 0,073 & 2.633 \\
\hline & Cruce de parámetros & 57,8777 & 16 & $-69,1318$ & 0,0297 & 2.802 & 145,973 & 16 & 19,9589 & 0,0755 & 2.633 \\
\hline \multirow[t]{7}{*}{ Educación } & Independencia & $1.038,7309$ & 9 & 968,559 & 0,2768 & 2.433 & 496,1887 & 9 & 425,5518 & 0,1726 & 2.562 \\
\hline & Endogamia constante (EC) & 262,1589 & 8 & 199,7838 & 0,1039 & 2.433 & 195,6969 & 8 & 132,9085 & 0,1028 & 2.562 \\
\hline & Endogamia diferenciada (ED) & 217,881 & 5 & 178,8966 & 0,0809 & 2.433 & 115,0139 & 5 & 75,7712 & 0,0623 & 2.562 \\
\hline & Cuasisimetría & 6,0254 & 3 & $-17,3653$ & 0,0063 & 2.433 & 4,9771 & 3 & $-18,5686$ & 0,0116 & 2.562 \\
\hline & Asociación uniforme con EC & 30,3891 & 7 & $-24,1891$ & 0,0369 & 2.433 & 15,9999 & 7 & $-38,9399$ & 0,021 & 2.562 \\
\hline & Asociación uniforme con ED & 9,4219 & 4 & $-21,7656$ & 0,0088 & 2.433 & 8,4672 & 4 & $-22,927$ & 0,0121 & 2.562 \\
\hline & Cruce de parámetros & 6,168 & 4 & $-25,0196$ & 0,0062 & 2.433 & 4,9944 & 4 & $-26,3998$ & 0,0117 & 2.562 \\
\hline \multirow[t]{5}{*}{ Religión } & Independencia & 708,5182 & 9 & 638,0015 & 0,2007 & 2.528 & $1.267,2637$ & 9 & 1196,5116 & 0,2563 & 2.595 \\
\hline & Endogamia constante (EC) & 69,255 & 8 & 6,5736 & 0,0546 & 2.528 & 112,0401 & 8 & 49,1493 & 0,0583 & 2.595 \\
\hline & Endogamia diferenciada (ED) & 2,9956 & 5 & $-36,1803$ & 0,0047 & 2.528 & 6,5772 & 5 & $-32,7295$ & 0,0047 & 2.595 \\
\hline & Cuasisimetría & 1,8511 & 3 & $-21,6545$ & 0,0047 & 2.528 & 4,4039 & 3 & $-19,1801$ & 0,0047 & 2.595 \\
\hline & Cruce de parámetros & 2,9428 & 4 & $-28,3979$ & 0,0046 & 2.528 & 5,9831 & 4 & $-25,4623$ & 0,0044 & 2.595 \\
\hline Posición & Independencia & 649,2962 & 9 & 577,2 & 0,1685 & 3.013 & 720,3456 & 9 & 649,2265 & 0,1835 & 2.703 \\
\hline \multirow[t]{4}{*}{ política } & Endogamia constante (EC) & 156,9023 & 8 & 92,8167 & 0,0726 & 3.013 & 161,2401 & 8 & 98,0231 & 0,0776 & 2.703 \\
\hline & Endogamia diferenciada (ED) & 74,1512 & 5 & 34,0978 & 0,0401 & 3.013 & 20,7538 & 5 & $-18,7567$ & 0,0203 & 2.703 \\
\hline & Cuasisimetría & 5,5347 & 3 & $-18,4974$ & 0,0091 & 3.013 & 5,2565 & 3 & $-18,4498$ & 0,0103 & 2.703 \\
\hline & Cruce de parámetros & 7,2525 & 4 & $-24,7903$ & 0,0133 & 3.013 & 13,0678 & 4 & $-18,5407$ & 0,0143 & 2.703 \\
\hline
\end{tabular}

Fuente: elaboración según ENACOES 2014. 
Cuadro 2C. Estadísticos de bondad de ajuste de modelo de cuasisimetría con endogamia diferenciada para encuestados, confidentes y tipo de lazo (familiar / no familiar)

\begin{tabular}{llrrrrrr}
\hline \multicolumn{1}{c}{ Variable } & \multicolumn{1}{c}{ Modelo } & & & & & & $\begin{array}{c}\text { Test de razón } \\
\text { de verosimilitud } \\
\text { M1 vs M2 }\end{array}$ \\
\hline Edad & Cuasisimetría sin interacción (M1) & 987,3870 & 45 & 600,3593 & 0,1672 & 5.435 & $\Delta G^{2}=899,51$ \\
& Cuasisimetría con interacción (M2) & 87,8742 & 25 & $-127,1412$ & 0,0364 & 5.435 & $p<0,001$ \\
\hline Educación & Cuasisimetría sin interacción (M1) & 213,8371 & 18 & 60,5456 & 0,0843 & 4.995 & $\Delta G^{2}=187,82$ \\
& Cuasisimetría con interacción (M2) & 26,0160 & 9 & $-50,6298$ & 0,016 & 4.995 & $p<0,001$ \\
\hline Religión & Cuasisimetría sin interacción (M1) & 85,8277 & 18 & $-67,9192$ & 0,0481 & 5.123 & $\Delta G^{2}=62,55$ \\
& Cuasisimetría con interacción (M2) & 23,1619 & 9 & $-53,7115$ & 0,0148 & 5.123 & $p<0,001$ \\
\hline Posición política & Cuasisimetría sin interacción (M1) & 60,7322 & 18 & $-94,9863$ & 0,0284 & 5.716 & $\Delta G^{2}=48,57$ \\
& Cuasisimetría con interacción (M2) & 12,1547 & 9 & $-65,7046$ & 0,0101 & 5.716 & $p<0,001$ \\
\hline
\end{tabular}

Fuente: elaboración propia según ENACOES 2014. 